\title{
Does surgery reduce knee osteoarthritis, meniscal injury and subsequent complications compared with non-surgery after $A C L$ rupture with at least 10 years follow-up? A systematic review and meta-analysis
}

\author{
Teodor Lien-Iversen (1) 1,2 Daniel Barklin Morgan, ${ }^{1,2}$ Carsten Jensen, ${ }^{1,2}$ \\ May Arna Risberg, ${ }^{3,4}$ Lars Engebretsen, ${ }^{3,4,5}$ Bjarke Viberg (i) 1,2
}

\begin{abstract}
- Additional material is published online only. To view, please visit the journal online (http://dx.doi.org/10.1136 bjsports-2019-100765)
\end{abstract}

'Department of Orthopaedic Surgery and Traumatology, Lillebaelt Hospital - University Hospital of Southern Denmark, Kolding, Denmark ${ }^{2}$ Institute of Regional Health Research, University of Southern Denmark, Odense, Denmark ${ }^{3}$ Department of Sport Medicine, Norwegian School of Sport Sciences, Oslo, Norway ${ }^{4}$ Division of Orthopedic Surgery, Oslo University Hospital, Oslo, Norway

${ }^{5}$ Institute of Clinical Medicine, Faculty of Medicine, University of Oslo, Oslo, Norway

\section{Correspondence to} Dr Bjarke Viberg, Department of Orthopaedic Surgery and Traumatology, Lillebaelt Hospital—University Hospital of Southern Denmark, Kolding 6000, Denmark

bjarke.viberg@rsyd.dk

Accepted 24 October 2019 Published Online First 15 November 2019

\section{ABSTRACT}

Objective We compared long-term follow-up from surgical versus non-surgical treatment of $A C L$ rupture regarding radiographic knee osteoarthritis $(O A)$, secondary surgery, laxity and patient-reported outcome measures (PROMs)

Design Systematic review and meta-analysis.

Data sources Embase, MEDLINE, CINAHL and the Cochrane Library databases.

Eligibility criteria for selecting studies Studies

directly comparing the minimally invasive surgical (arthroscopy or miniarthrotomy) and non-surgical treatment of $A C L$ rupture with at least 10 years of follow-up in adult patients were included.

Results Five studies met the eligibility criteria. A metaanalysis revealed a higher risk of radiographic knee $\mathrm{OA}$ and a lower risk of secondary meniscal surgery for patients in the surgical group. The risk of graft rupture/ secondary $A C L$ revision and secondary $A C L$ reconstruction was equal in the surgical and non-surgical groups. Knee laxity was lower among patients in the surgical group in four studies. No difference was found in the PROMs (ie, International Knee Documentation Committee, Tegner, Knee Injury and Osteoarthritis Outcome, and Lysholm scores).

Conclusion The risk of radiographic knee OA was higher, but the risk of secondary meniscal injury was lower 10 years after surgical treatment of $A C L$ rupture. The risk of graft rupture/secondary $A C L$ revision or secondary reconstruction was unrelated to treatment type. The degree of knee laxity was reduced after surgical treatment in comparison with non-surgical treatment, while PROMs were similar. However, due to the methodological challenges highlighted in this systematic review, these findings must be interpreted with caution. PROSPERO registration number CRD42019119468

\section{INTRODUCTION}

ACL rupture can be treated surgical or nonsurgical. ${ }^{1}$ Recent high-quality comparative studies using midterm follow-up have largely failed to show any clear advantage of surgical versus non-surgical treatment on knee osteoarthritis (OA) development and patient-reported outcome measures (PROMs). ${ }^{2}{ }^{3}$ Prior systematic reviews with longterm follow-up also lack evidence to support either surgical or non-surgical treatment. ${ }^{4-6}$ However, a major problem with these comparative studies is the considerable number of patients who initially received non-surgical treatment but later opted for surgical treatment and thereby make the consequences of the initial treatment harder to track. ${ }^{278}$ Another shortcoming of the existing comparative studies is selection bias, since patients with worse injuries (eg, concomitant ligament, cartilage and meniscal injuries) are generally initially treated with surgery.

Open ACL reconstruction is rarely performed today, and minimally invasive techniques are dominant in modern clinical practice. ${ }^{9}$ Most systematic reviews with long-term follow-up include studies with open ACL reconstruction treatment, ${ }^{4} 51011$ thereby limiting the generalisability and clinical relevance of the findings. Consequently, excluding open ACL reconstruction studies from this review will provide a more up-to-date picture.

The aim of this study was to systematically review the literature and compare minimally invasive surgical (ie, arthroscopy or miniarthrotomy) versus non-surgical treatment in patients with ACL rupture who had at least 10 years of follow-up concerning the severity of radiographic knee OA, secondary ACL surgery and meniscectomy, knee laxity and PROMs.

\section{METHODS}

\section{Protocol and registration}

This systematic review was performed according to the Preferred Reporting Items for Systematic Review and Meta-Analysis guidelines. ${ }^{12}$ The protocol was registered in the international prospective register of systematic reviews, PROSPERO, and met all the eligibility criteria for protocol registration.

\section{Eligibility criteria}

The PICO was defined as primary ACL rupture in adults treated with either surgical or non-surgical treatment with a minimum of 10 years of follow-up and radiographic knee OA, secondary surgery, laxity and PROMs data.

Studies were included if they:

- Included a comparison of surgical and nonsurgical treatment of ACL rupture.

- Covered the use of a minimally invasive surgical technique (arthroscopic or miniarthrotomy).

- Included a minimum of 10 years of follow-up.

- Included a patient mean age of $\geq 18$ years. Studies were excluded if they: 
- Were animal or cadaveric studies.

- Were articles not in the English, German or Scandinavian languages.

- Included patients with prior major knee surgery.

Published studies with levels of evidence I-IV were included with the exception of population-based cohort studies. Editorials and conference abstracts were also excluded. Arthroscopic verification of ACL tear was not considered a surgical intervention.

\section{Information sources and literature search}

A literature search was performed in October 2018 among four electronic databases: Embase, MEDLINE, CINAHL and the Cochrane Library. In addition, the reference lists of relevant studies were screened for additional eligible articles. The search strategy included the key term 'anterior cruciate ligament' in combination with surgical and non-surgical treatment search terms as well as outcomes. For the purpose of including all relevant literature, a sensitive search strategy was used:

(Anterior cruciate ligament OR ligamentum cruciatum anterius) AND ((surgery OR operation OR reconstruction OR reconstructions $O R$ reconstructive $O R$ transplantation $O R$ allograft $O R$ allografts $O R$ replacement $O R$ autograft $O R$ autografts OR graft OR grafts) OR (operation OR non-surgery OR exercise OR rehabilitation OR non-operative)) AND (osteoarthritis OR reoperation OR complications OR complication OR activities of daily living OR deep infection OR deep infections OR patientreported outcome measures OR PROM OR patient-reported outcome OR removal of graft OR adjacent fracture OR knee arthroplasty OR total knee arthroplasty OR meniscal surgery OR KOOS OR Tegner OR Lysholm OR WOMAC OR IKDC OR KUJALA)

Librarians at the University Library of Southern Denmark assisted in shaping and optimising the search strategy.

\section{Study selection}

Search results were extracted to EndNote (Clarivate Analytics, Philadelphia, Pennsylvania, USA), duplicates were removed and the remaining articles were imported into Covidence (Veritas Health Innovation, Melbourne, Australia). Titles and abstracts were screened by two independent reviewers (TL-I and DBM). The full versions of potential articles were read to determine eligibility. In case of multiple studies using the same cohort data, the studies with longer follow-up time and primary focus on tibiofemoral OA were preferred and included. Conflicts were resolved with discussion among the review team (TL-I, DBM, $\mathrm{CJ}$ and $\mathrm{BV})$.

\section{Data collection}

Data extraction was performed independently by the two aforementioned reviewers (TL-I and DBM) and cross-checked for errors. A data extraction sheet was created for the purpose. Discrepancies were resolved by consensus.

\section{Data items}

The extracted data included: sex; age; body mass index (BMI); country; Tegner activity level ${ }^{13}$; time from injury to intervention; meniscal and/or chondral injuries; knee laxity as measured by a KT-1000 arthrometer ${ }^{14}$; secondary injuries and/ or surgical interventions; surgical intervention (arthroscopically or miniarthrotomy surgery, graft type and rehabilitation after surgery), non-surgical intervention (rehabilitation) and measurements of knee OA, specifically radiographic, Knee Injury and
Osteoarthritis Outcome Score (KOOS), ${ }^{15}$ International Knee Documentation Committee (IKDC) subjective score $^{16}$ and Lysholm scoring scale values. ${ }^{17}$

Secondary interventions were defined as: ACL reconstruction in the non-surgical group and ACL revision or graft rupture in the surgical group. These procedures are comparable as they are the manifestation of treatment failure of the primary intervention in both groups. Minimally invasive surgical treatment was defined as ACL reconstruction using either arthroscopically or miniarthrotomy technique.

Cut-off values for knee OA for the different radiographic classification systems were, in accordance with prior studies, ${ }^{46} 18$ defined as: Kellgren and Lawrence system ${ }^{19}$ grade $\geq 2$; IKDC qualification $^{20}$ grade $\geq \mathrm{C}$; and for the Osteoarthritis Society Research International (OARSI) classification system, ${ }^{21}$ a joint space narrowing (JSN) of grade 2, sum of marginal osteophyte grades $\geq 2$ or a grade $1 \mathrm{JSN}$ in combination with a grade 1 marginal osteophyte.

\section{Risk of bias in individual studies}

A methodological quality appraisal of the studies was performed using the Downs and Black checklist. ${ }^{22}$ The Downs and Black checklist is a frequently employed method to evaluate reporting, external validity, internal validity, confounding, bias and statistical power that has been recognised as a comprehensive and suitable option for assessing systematic reviews in the appraisal of both non-randomised and randomised controlled trials. ${ }^{23}$ In the present investigation, the item concerning sufficient power was modified to whether the study presented a sample size calculation or not. It was modified because there were insufficient data to make an adequate power calculation. Items received 1 point if the criterion was met and 0 points if not (one item could score two points). Zero points were given if the item was unable to be quantified. The lowest methodological quality score possible was 0 points and the highest was 28 points. The two reviewers performed an appraisal of the studies. Any disagreements were solved by discussion.

\section{Summary measures and synthesis of results}

The outcomes measures were reported by study and metaanalysed using forest plots using RevMan V.5.3 (the Nordic Cochrane Center, Copenhagen, Denmark). The intervention effect was expressed as a risk ratio (RR) including a 95\% CI. Statistical significance was defined as $\mathrm{p} \leq 0.05$. Pooled data were assessed for heterogeneity using the $\chi^{2}$ and $\mathrm{I}^{2}$ tests. Heterogeneity was defined as 'absent' (0\%-25\%), 'low' (26\%-50\%), 'moderate' (51\%-75\%) or 'high' (76\%-100\%). A fixed effects meta-analysis was performed when the $\mathrm{I}^{2}$ test outcome was less than $50 \%$.

\section{RESULTS}

\section{Study selection}

In total, 10401 studies were initially included. Of those, 10364 were excluded based on a review of their titles and abstracts, and the full versions of the remaining 37 studies were assessed for further eligibility (figure 1). During the full-text review, an additional 32 studies were excluded for the following reasons: open surgery $(n=12)$, not comparing surgery with non-surgery $(n=8)$, conference abstracts $(n=5)$, repeated study cohort $(n=3),{ }^{24-26}$ less than 10 years of follow-up $(n=2)$ and population-based cohort studies $(n=2)$. The remaining five studies ${ }^{727-29}$ fulfilled all of the eligibility criteria and so were included in this review. 

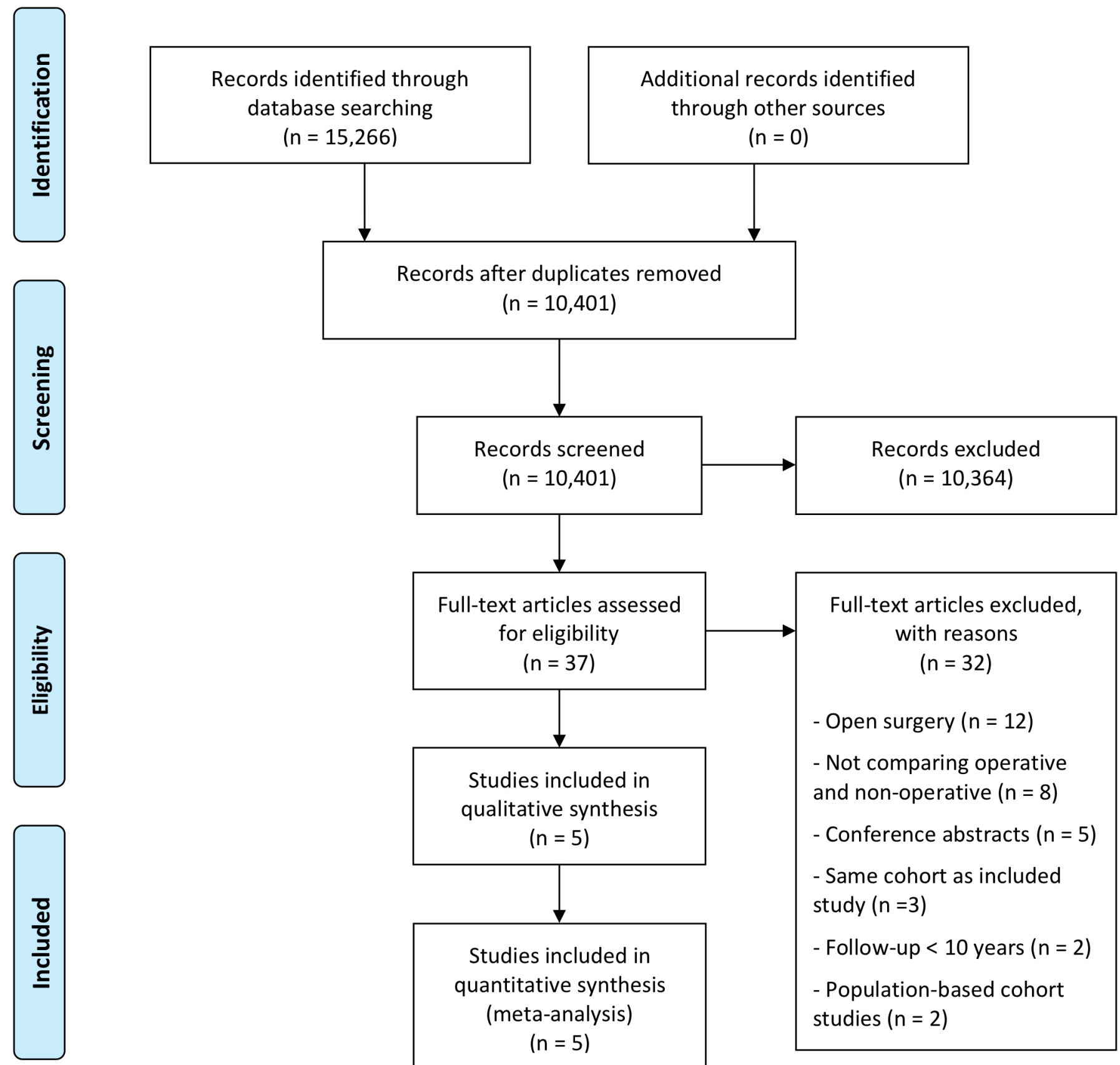

Figure 1 PRISMA flow diagram of search strategy. PRISMA, Preferred Reporting Items for Systematic Review and Meta-Analysis.

\section{Study characteristics}

Of the five included studies, two were prospective, ${ }^{27} 28$ including one that was randomised, ${ }^{28}$ and three studies were retrospective. ${ }^{7829}$ A total of 371 patients were included, with a distribution of 164 surgically and 207 non-surgically treated patients (table 1). Follow-up ranged from 10 to 20 years in length. All ACL ruptures were confirmed by MRI, ${ }^{28}$ arthroscopy $^{7827}$ or either one. ${ }^{29}$ One study ${ }^{29}$ included a unique subgroup of patients-that is, high-level athletes defined by Tegner score of more than 7 points (median of 9 points). All retrospective studies were pair-matched with respect to age and sex of the included patients. Other matching factors applied included BMI, follow-up duration and concomitant injuries. A summary of the study and patient characteristics is presented in table 1 .
Risk of bias in individual studies

Methodological quality was evaluated using the Downs and Black checklist, with $0-28$ points being possible but with the included studies ranging only from $14^{7}$ to 18 points, ${ }^{8} 2729$ with a mean score of 16.8 points (table 1 ). Collectively, the studies achieved the highest scores for items 1-10 covering reporting. However, for items 21-27, concerning confounding/selection bias, the studies attained considerably lower scores. The specific scores given for the studies are available in online supplementary appendix 1 .

\section{Radiographic knee OA}

All the five studies measured the severity of radiographic knee OA using either the Kellgren and Lawrence system, ${ }^{29}$ IKDC grading $^{828}$ or the OARSI atlas. ${ }^{27}$ The prevalence of OA ranged 
Table 1 Study and patient characteristics

\begin{tabular}{|c|c|c|c|c|c|c|c|c|c|c|c|}
\hline \multirow[b]{2}{*}{ Study } & \multirow[b]{2}{*}{ Country } & \multirow[b]{2}{*}{ Study design } & \multirow{2}{*}{$\begin{array}{l}\text { Follow-up } \\
\text { (years) }\end{array}$} & \multirow{2}{*}{$\begin{array}{l}\text { Quality } \\
(0-28)\end{array}$} & \multirow{2}{*}{$\begin{array}{l}\text { Time, injury to } \\
\text { surgery }\end{array}$} & \multicolumn{2}{|l|}{$\mathrm{n}$} & \multicolumn{2}{|c|}{$\begin{array}{l}\text { Mean age (SD) at follow- } \\
\text { up, years }\end{array}$} & \multicolumn{2}{|c|}{$\operatorname{Sex}(m / f)$} \\
\hline & & & & & & $S$ & NS & $S$ & NS & $S$ & NS \\
\hline Kessler et al ${ }^{7}$ & Switzerland & Retrospective & 11 & 14 & $\mathrm{~N} / \mathrm{A}$ & 60 & 49 & $30.7(12.5-54)$ & & $68 / 41+$ & \\
\hline Streich et $a l^{8}$ & Germany & Retrospective & 15 & 18 & 7.3 months $\ddagger$ & 40 & 40 & $26.0(6.4)^{*}$ & $24.0(6.5)^{*}$ & $28 / 12$ & $28 / 12$ \\
\hline Tsoukas et al ${ }^{28}$ & Greece & Prospective randomised & 10 & 16 & 6 weeks $\ddagger$ & 17 & 15 & $31(20-36) \ddagger$ & $33(25-39) \ddagger$ & $17 / 0$ & $17 / 0$ \\
\hline
\end{tabular}

Quality is measured by Downs and Black checklist.

${ }^{*}$ Reported at injury.

tReported for combined participants in the study.

$\ddagger$ Range.

f, female; m, male; N/A, not available; NS, non-surgical group;S, surgical group.

from $24 \%^{28}$ to $80 \%{ }^{29}$ in the surgical groups and $11 \% \%^{27}$ to $68 \%{ }^{29}$ in the non-surgical groups (table 2). A significantly lower prevalence of radiographic knee OA in favour of the non-surgical groups was shown in two studies $\left(p=0.03\right.$ in both). ${ }^{72}$ The meta-analysis revealed that the risk of radiographic knee OA was higher in the surgical groups (RR 1.42 (95\% CI 1.09 to 1.85$)$ ) (figure 2).

\section{Secondary surgical interventions}

Graft ruptures, secondary ACL reconstructions and meniscectomies were reported in four studies (table 3). ${ }^{782829}$ Due to the study design for one investigation, the surgical group consisted of patients from the non-surgical group who had undergone secondary ACL reconstruction ${ }^{27}$ and were not included in the analysis of secondary surgery. Only one study ${ }^{29}$ differentiated between lateral and medial meniscectomy. Two ${ }^{78}$ of four studies $^{783031}$ found a significantly reduced need for secondary meniscectomy in the surgical group as compared with the nonsurgical group (both $\mathrm{p}<0.03$ ). Our meta-analysis revealed that the risk of graft rupture or secondary ACL revision was independent of treatment (RR 0.90 (95\% CI 0.49 to 1.66)) (figure 2). Separately, the risk of secondary meniscectomy was reduced significantly in patients who had surgical treatment (RR 0.34 (95\% CI 0.20 to 0.58$)$ ) (figure 2).

\section{Knee laxity}

Knee laxity (side-to-side difference) was measured using a KT-1000 arthrometer in all studies. ${ }^{7}$ 27-29 $^{27}$ Knee laxity ranged from 1.5 to $5.3 \mathrm{~mm}$ in the surgical groups versus 2.1 to $5.7 \mathrm{~mm}$ in the non-surgical groups. However, one study ${ }^{29}$ reported the number of patients with a side-to-side difference of more than $3 \mathrm{~mm}$ was 10 of $25(40 \%)$ in the surgical group and 19 of 25 $(76 \%)$ in the non-surgical group $(p=0.013)$. Still, four of the five studies found significantly less knee laxity in the surgical group. $^{727-29}$

\section{Patient-reported outcome measures}

IKDC subjective score was reported in three studies, 82829 with one study reporting better scores for patients in the surgical group $(p=0.04)$ (table 4$).^{28}$ The KOOS score was reported in two studies, ${ }^{27} 29$ wherein the non-surgical group reported a significantly better score on the pain subscale than compared with the surgical group in one study $(\mathrm{p}=0.35),{ }^{27}$ while there was no significant difference between the two groups in the other study. $^{29}$

\section{Intervention description}

Surgical intervention was performed within a period of 6 weeks $^{28}$ to 4 years $^{27}$ after the initial injury. Arthroscopic surgical technique was performed in four studies ${ }^{7} 82829$ and miniarthrotomy in one. ${ }^{27}$ Bone-patellar tendon-bone was the preferred graft for ACL reconstruction, ${ }^{7} 2729$ whereas reconstruction with four-strand semitendinosus-gracilis graft was performed in one study. ${ }^{28}$ All surgically treated patients in the five studies participated in exercise-based rehabilitation programmes of various length and two studies used supervised physiotherapy. ${ }^{727}$

In the non-surgical group, all of the studies used physiotherapysupervised rehabilitation, except for one subgroup in one study. ${ }^{27}$ Non-surgical treatment was initiated shortly following the confirmation of diagnosis. Follow-up time for the rehabilitation was only reported in two studies. ${ }^{27}$ Instructions to gradually return to more strenuous physical activities were given to patients in both groups.

Table 2 Radiographic knee osteoarthritis (OA)

\begin{tabular}{|c|c|c|c|c|c|c|c|}
\hline \multirow[b]{2}{*}{ Study } & \multirow[b]{2}{*}{ Scoring system } & \multicolumn{3}{|c|}{ Surgical } & \multicolumn{3}{|c|}{ Non-surgical } \\
\hline & & $\mathrm{n}$ & Grades & $\mathrm{OA}(\%)$ & $\mathrm{n}$ & Grades & $O A(\%)$ \\
\hline Kessler et al ${ }^{7}$ & K\&L & 60 & $27 / 6 / 25 / 2 / 0$ & $27(45)$ & 49 & $30 / 7 / 10 / 2 / 0$ & $12(24)$ \\
\hline Neuman et $a l^{27}$ & OARSI & 17 & $N / A$ & $6(35)$ & 62 & $\mathrm{~N} / \mathrm{A}$ & $7(11)$ \\
\hline Streich et $a l^{8}$ & IKDC & 40 & $14 / 11 / 3 / 12$ & $15(38)$ & 40 & $18 / 7 / 4 / 11$ & $15(38)$ \\
\hline Tsoukas et al ${ }^{28}$ & IKDC & 17 & $\mathrm{~N} / \mathrm{A}$ & $4(24)$ & 15 & $\mathrm{~N} / \mathrm{A}$ & $5(33)$ \\
\hline van Yperen et al/ & K\&L & 25 & $1 / 4 / 16 / 3 / 0 /(1)$ & $20(80)$ & 25 & $3 / 5 / 12 / 4 / 1 /(0)$ & $17(68)$ \\
\hline
\end{tabular}

K\&L: 0/1/2/3/4/(total knee arthroplasty) and IKDC: A/B/C/D.

IKDC, International Knee Documentation Committee; K\&L, Kellgren and Lawrence; N/A, not available; OARSI, Osteoarthritis Society Research International. 
Radiographic knee osteoarthritis

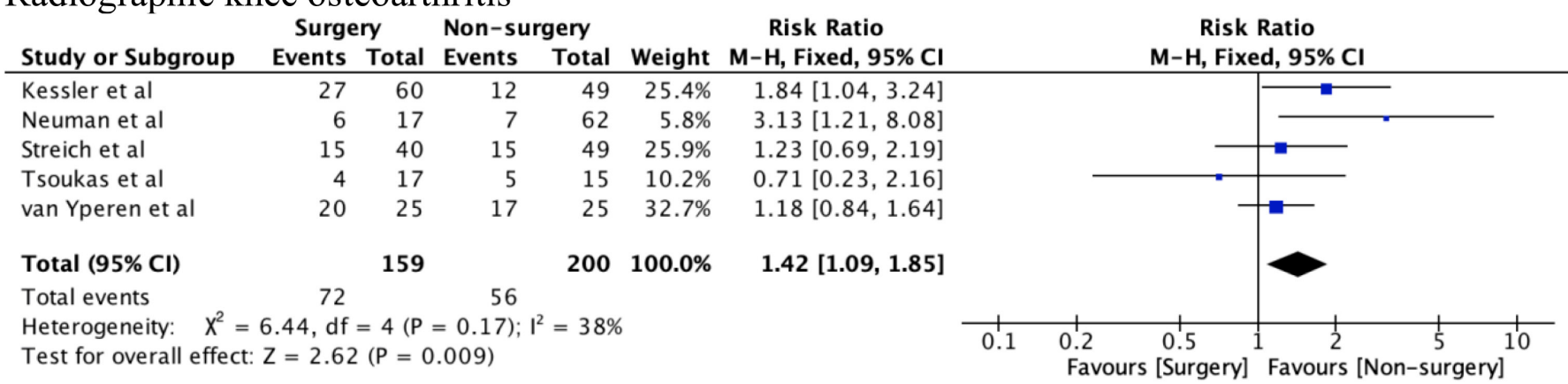

Graft rupture or subsequent ACL reconstruction

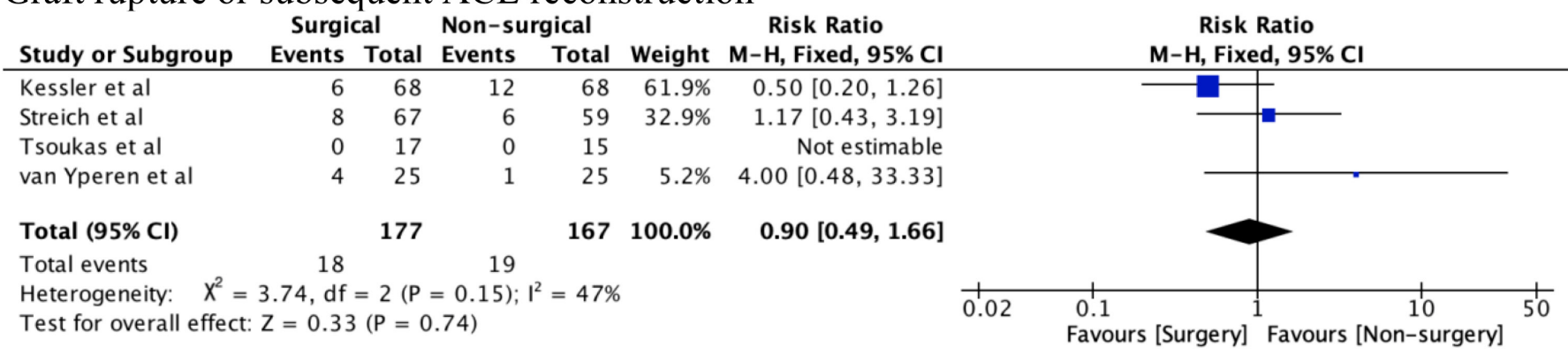

Subsequent meniscectomy

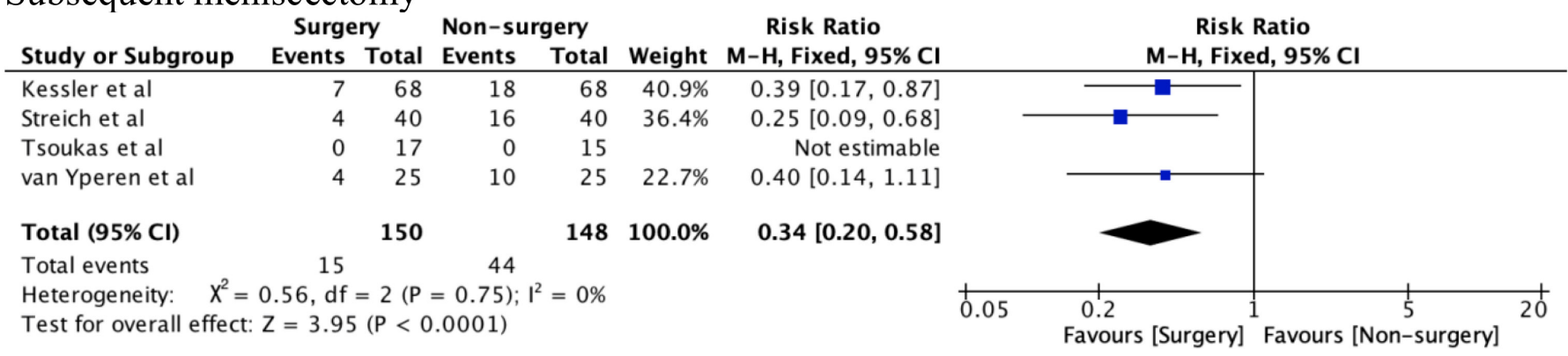

Figure 2 Meta-analysis.

\section{DISCUSSION}

This systematic review included five studies comparing surgical versus non-surgical treatment of ACL rupture with more than 10 years of follow-up. Two studies were prospective $e^{27} 28$ and three were retrospective. ${ }^{7}{ }^{29}$ Methodological shortcomings were evident in all studies, as demonstrated by a mean quality score of 16.8 points out of 28 points possible.

Based on the available data of 164 surgically and 207 conservatively treated patients, the risk of radiographic knee OA was higher in the surgical group than in the non-surgical group. The risk of secondary ACL reconstruction was independent of treatment, whereas secondary meniscectomies were performed significantly less frequently in the surgical group. Patients who underwent ACL reconstruction experienced significantly less knee laxity. The PROMs (ie, Lysholm, IKDC, Tegner and KOOS scores) were independent of group allocation.

Radiographic knee OA

Our meta-analysis revealed a higher risk of knee OA in patients who had gone through surgery in comparison with those treated via rehabilitation alone.

However, caution must be applied in the interpretation and conclusions of this meta-analysis. With the exception of the one

Table 3 Secondary surgical interventions

\begin{tabular}{|c|c|c|c|c|}
\hline & \multicolumn{2}{|l|}{ Surgical } & \multicolumn{2}{|l|}{ Non-surgical } \\
\hline & Graft rupture or ACL revision (\%) & Meniscectomy (\%) & $\mathrm{ACL}$ reconstruction $(\%)$ & Meniscectomy (\%) \\
\hline Kessler et al ${ }^{7}$ & $6 / 68(9)^{*}$ & $7 / 68(10)^{*}$ & $12 / 68(18)^{*}$ & $18 / 68(26)^{*}$ \\
\hline Tsoukas et al ${ }^{28}$ & $0 / 17(0)$ & $0 / 17(0)$ & $0 / 15(0)$ & $0 / 15(0)$ \\
\hline van Yperen et a $\left.\right|^{29}$ & $4 / 25(16)$ & $4 / 25(16)$ & $1 / 25(4)$ & $10 / 25(40)$ \\
\hline
\end{tabular}


Table 4 Patient-reported outcome measures

\begin{tabular}{|c|c|c|c|c|c|c|c|c|}
\hline \multirow[b]{3}{*}{ Study } & \multicolumn{4}{|l|}{ Surgical } & \multicolumn{4}{|l|}{ Non-surgical } \\
\hline & \multirow[b]{2}{*}{ IKDC subj } & \multirow[b]{2}{*}{ Lysholm } & \multicolumn{2}{|l|}{ Tegner } & \multirow[b]{2}{*}{ IKDC subj } & \multirow[b]{2}{*}{ Lysholm } & \multicolumn{2}{|l|}{ Tegner } \\
\hline & & & BL & FU & & & BL & FU \\
\hline Kessler et al ${ }^{7}$ & $\mathrm{~N} / \mathrm{A}$ & $\mathrm{N} / \mathrm{A}$ & 5.4 & 5.3 & N/A & N/A & 5.9 & 4.9 \\
\hline Neuman et $a l^{27}$ & $\mathrm{~N} / \mathrm{A}$ & $86(16)$ & $7^{*}$ & 4.5 & $\mathrm{~N} / \mathrm{A}$ & $82(19)$ & $7^{*}$ & 3.7 \\
\hline Streich et $a l^{8}$ & $69.9(17.0)$ & $68.0(19.8)$ & $7.6(1.5)$ & $4.7(1.8)$ & $75.9(13.1)$ & 75.5 (15.9) & $7.1(1.4)$ & $5.1(1.9)$ \\
\hline Tsoukas et al ${ }^{28}$ & $86.7(6.5)$ & $\mathrm{N} / \mathrm{A}$ & $7(5-7)^{*}$ & $7(5-7)^{*}$ & $77.5(13)$ & $\mathrm{N} / \mathrm{A}$ & $7(4-7)^{*}$ & $5(3-7)^{*}$ \\
\hline Van Yperen et a ${ }^{29}$ & $81.6(59.8-89.1) \dagger$ & $86.0(75.5-91.0) \dagger$ & $9(7-9) \dagger$ & $5(3-6) \dagger$ & $78.2(61.5-92.0) \dagger$ & $89.0(75.5-95.5) \dagger$ & $9(7-9) \dagger$ & $5(4-6) \dagger$ \\
\hline
\end{tabular}

Data are presented as mean (SD) unless otherwise specified.

*Median (range).

†Median (IQR).

BL, baseline; FU, follow-up;IKDC subj, International Knee Documentation Committee subjective form; N/A, not available.

randomised study, ${ }^{28}$ the patients treated surgically had more subjective knee instability preoperatively compared with those treated non-surgically. The choice of treatment was based on the patient's wishes as well as the treating surgeons' advice. In two studies, ${ }^{27} 29$ patients who did not respond well to non-surgical treatment underwent ACL reconstruction and were, therefore, included in the surgery group. In the decision-making process, the surgeons' guidance may have influenced patients' choice of treatment, as we do not know in what way the surgeons gave their advice, as the use of a valid shared-decision tool was not reported. Marx et $a l^{30}$ found that American orthopaedic surgeons consider several factors when making a decision in favour of surgical treatment, as follows: giving way in daily activities, giving way in sporting activities, high-demand activity, recurrent swelling, radiographic knee OA and repairable meniscal tear. These findings suggest a general tendency towards treating the most extensive ACL injuries surgically, thus introducing a potential risk of selection bias and thereby skewing the results in favour of non-surgical treatment. Earlier literature suggests that high-level pivoting sports and a higher activity level over time can lead to an increase in $\mathrm{OA},{ }^{31}$ possibly promoting more knee $\mathrm{OA}$ in the surgical group. This, however, is disputed by more recent literature, wherein opposite findings indicate that those who are more physically active and who returned to pivoting sports had better knee function and less radiographic knee OA. ${ }^{32}$ Streich et $a l^{8}$ and Kessler et $^{\mathrm{al}} \mathrm{l}^{7}$ both excluded patients who received secondary ACL reconstruction. Kessler et $a l^{7}$ excluded patients receiving both primary and secondary meniscectomies. In this study, more than double the number of patients was excluded from the nonsurgical group than the surgical group, thereby possibly skewing the outcomes of the groups during follow-up. Hence, a possible underestimation of knee OA in the remaining patients in the nonsurgical group could have occurred. This is also indicated by the methodological quality assessment, wherein the studies collectively had low scores in the items on confounding/selections bias. Conducting a randomised controlled trial (RCT) could help to solve this problem. However, this is difficult to complete due to ethical considerations and recruiting, as reported by Frobell et $a l^{33}$ who conducted the only RCT to date with 5 years follow-up comparing early rehabilitation and ACL reconstruction to rehabilitation and optional delayed ACL reconstruction. ${ }^{2}$ A discrepancy is present when comparing this study's results to our meta-analysis, as Frobell et al did not report more radiographic knee OA in the surgical group. ${ }^{2}$ A possible explanation for this variance is the shorter follow-up period of 5 years in Frobell et al's study versus the 10 -year or more period in ours. ${ }^{2}$ This difference supports the assumption of the aforementioned selection bias of the included studies in this systematic review.
The reasoning behind choosing non-surgical treatment is that neuromuscular and strength training can stabilise the knee by way of increased muscle strength and enhanced proprioception. ${ }^{34}$ With one exception, ${ }^{27}$ none of the included studies reported follow-up of physiotherapy treatment for more than 3 months. Likewise, the compliance rate for these patients remains unknown. This could result in an underestimation of the potential beneficial effect of consistent physiotherapy treatment.

All patients in the study by Neuman $e t \mathrm{al}^{27}$ with radiographic knee OA at follow-up had primary or secondary meniscectomy. A tendency was confirmed by van Yperen et al, ${ }^{29}$ who found that $94 \%$ in the surgical group and $68 \%$ in the non-surgical group, among those who underwent meniscectomy, developed knee OA. These findings correspond to the findings of Øiestad et $a l,{ }^{4}$ who, in a large systematic review, identified a significantly higher prevalence of ACL injuries with concomitant meniscal injury versus without (0\%-13\% compared with 21\%-48\%). This underlines the fact that meniscal injury and meniscectomy are important risk factors for developing knee OA.

The only study with two follow-up time points, by van Yperen et $a l,{ }^{29}$ showed that radiographic knee OA developed in 19 of 50 $(38 \%)$ patients at 10 years of follow-up and in 37 of $50(74 \%)$ patients at 20 years of follow-up. This corresponds with findings of a recent meta-analysis by Cinque et al, ${ }^{35}$ who showed that the prevalence of post-traumatic radiographic knee OA developed significantly at 5, 10 and 20 years after surgical treatment is as follows: $11 \%, 21 \%$ and $52 \%$, respectively. Long-term follow-up is, therefore, necessary to examine the real late consequences of ACL rupture.

The presence of radiographic knee OA in this review was determined by cut-off values used in three different classification systems. Two studies using the Kellgren and Lawrence approach $^{729}$ and one using OARSI ${ }^{27}$ classified more patients with knee $\mathrm{OA}$ in the surgical groups, while two studies using IKDC $^{828}$ found no difference between the groups. There are challenges that appear when comparing results among studies that employed different classification systems. For example, Culvenor et $a l^{18}$ determined that when using the OARSI classification system, radiographic knee OA was nearly two times as common as when the Kellgren and Lawrence classification was used. Likewise, in the study by the MARS group, ${ }^{36}$ differences in interobserver reliability were present (IKDC versus Kellgren and Lawrence). Due to the limited number of studies, it was not possible to include studies using only a single classification system.

The findings of this review do not conclude on whether surgical or non-surgical treatment is preferable based on the patient-reported outcomes. There were no associations between 
increased risk of radiographic knee OA and PROMs in the surgical group. These observations are similar to earlier findings by Barker $e t a l,{ }^{37}$ who found no association between radiographic knee OA and PROMs.

Our findings also seem to be somewhat consistent with those of Øiestad et $a l,^{38}$ who identified no significant association between radiographic OA and all subscales of KOOS, with the exception of KOOS symptoms. They did, however, find significantly more symptoms among those with severe radiographic knee OA.

\section{Secondary surgical intervention}

Our meta-analysis revealed patients presented a significantly lower risk of having secondary meniscal surgery when initially treated surgically. However, the risk of having secondary ACL injury or surgery was not highly different between the two groups. This is in contrast to earlier findings by Chalmers et $a l,{ }^{6}$ who, in their meta-analysis, found that surgical patients had less need of secondary ACL surgery. In a similar fashion, Sanders $e{ }^{a} l^{39}$ found in their register-based study, with a mean follow-up of 14 years, that surgically treated patients had a significantly lower risk of experiencing secondary symptomatic knee OA, meniscal tear and total knee alloplasty versus nonoperatively treated patients. This could explain why we did not find a similar trend as that seen by Sanders et al and Chalmers et al. Selection bias could have resulted in an overestimation of secondary graft rupture in the surgical group, compared with the non-surgical group, as the young and highly physically active patients most often receive surgical treatment, especially those who want to return to participation in high-level pivoting sports.

\section{Limitations and strengths}

There were several limitations to our study. The five studies included did not measure radiographic OA with the same scoring system, making comparisons difficult. In a similar fashion, the studies used different PROMs. Surgical interventions also differed regarding specific technical details. Likewise, non-surgical (eg, physiotherapy, bracing and non-treatment) approaches were not equal across studies with respect to follow-up time points, programme content and supervision. Furthermore, no studies reported specific compliance rates with rehabilitation programmes. The inclusion of a larger number of patients would have been preferable. Publication bias may have influenced the authors' reporting in various studies. Lastly, the studies included in this systematic review included populations from European countries only, resulting in more homogenous patient groups and possibly reducing generalisability to the other parts of the world.

Notwithstanding these limitations, a strength of this review is that all reconstructive surgeries in our studies were performed by arthroscopy or miniarthrotomy, minimally invasive techniques that are comparable and used in today's practice. Only studies directly comparing surgical and non-surgical treatment were included. Also, two independent reviewers conducted the systematic literature search, study selection and data extraction.

Future studies should focus on limiting bias, preferably by conducting randomised clinical trials. Although difficult to achieve, non-randomised studies should try to reduce the inherent bias of patients with more symptoms being treated surgically. Longer follow-up periods could help to establish the two treatments' association with knee OA over a lifespan. Measurements of OA, both radiographic and subjective, should be similar.

\section{CONCLUSION}

The risk of radiographic knee OA was higher, but the risk of secondary meniscal injury was lower 10 years after surgical treatment of ACL rupture. The risk of graft rupture/secondary ACL revision or secondary reconstruction was unrelated to treatment type. The degree of knee laxity was reduced after surgical treatment in comparison with non-surgical treatment, while patient-reported outcomes were similar. However, due to the methodological challenges highlighted in this systematic review, these findings must be interpreted with caution.

\section{What is already known}

- $\mathrm{ACL}$ rupture is a common injury that is associated with an increased risk for osteoarthritis (OA) later in life following either surgical or non-surgical treatment.

- Young and physically active individuals comprise the patient group who predominantly experience $\mathrm{ACL}$ ruptures.

- Even though some literature suggests that surgical and non-surgical treatment are somewhat equal in regard to $\mathrm{OA}$ and patient-reported outcome measures, it is unclear which treatment strategy is best in the long-term (>10 years).

\section{What are the new findings}

- There is a lack of high-quality studies comparing minimally invasive $\mathrm{ACL}$ surgery with non-surgical treatment of $\mathrm{ACL}$ rupture with long-term follow-up.

- Surgically treated patients have a higher risk of experiencing osteoarthritis in the knee versus non-surgically treated patients.

- More non-surgically treated patients underwent secondary meniscal surgery as compared with those who received primary surgical treatment after their $\mathrm{ACL}$ rupture.

- The need for secondary ACL surgery was equal between the surgical and non-surgical groups.

\section{Twitter Lars Engebretsen @larsengebretsen}

Contributors BV conceived the project, while BV and CJ led the design (intervention selection, patient population, data management and statistical analyses). TL-I, DBM, MAR and LE contributed to the design. TL-I and DBM performed the literature search, extraction of data, quality assessment of studies and synthesis of the results, and drafted the manuscript. All the authors provided critical intellectual input to the manuscript and read and approved the final version of the manuscript, agreeing to be accountable for all aspects of the work.

Funding The authors have not declared a specific grant for this research from any funding agency in the public, commercial or not-for-profit sectors.

Competing interests BV reports personal fees for lectures from Zimmer Biomet and Osmedic Swemac outside the submitted work.

\section{Patient consent for publication Not required.}

Provenance and peer review Not commissioned; externally peer reviewed.

\section{ORCID iDs}

Teodor Lien-Iversen http://orcid.org/0000-0002-0462-4588

Bjarke Viberg http://orcid.org/0000-0001-5169-4282

\section{REFERENCES}

1 Rahr-Wagner L, Lind M. The Danish knee ligament reconstruction registry. Clin Epidemiol 2016:8:531-5.

2 Frobell RB, Roos HP, Roos EM, et al. Treatment for acute anterior cruciate ligament tear: five year outcome of randomised trial. BMJ 2013;346:f232. (no pagination).

3 Wellsandt E, Failla MJ, Axe MJ, et al. Does anterior cruciate ligament reconstruction improve functional and radiographic outcomes over Nonoperative management 5 years after injury? Am J Sports Med 2018;46:2103-12. 
4 Øiestad BE, Engebretsen L, Storheim K, et al. Knee osteoarthritis after anterior cruciate ligament injury: a systematic review. Am J Sports Med 2009;37:1434-43.

5 Smith TO, Postle K, Penny F, et al. Is reconstruction the best management strategy for anterior cruciate ligament rupture? A systematic review and meta-analysis comparing anterior cruciate ligament reconstruction versus non-operative treatment. Knee 2014;21:462-70.

6 Chalmers PN, Mall NA, Moric M, et al. Does ACL reconstruction alter natural history?: a systematic literature review of long-term outcomes. J Bone Joint Surg Am 2014;96:292-300.

7 Kessler MA, Behrend $\mathrm{H}$, Henz S, et al. Function, osteoarthritis and activity after ACLrupture: 11 years follow-up results of conservative versus reconstructive treatment. Knee Surg Sports Traumatol Arthr 2008;16:442-8.

8 Streich NA, Zimmermann D, Bode G, et al. Reconstructive versus non-reconstructive treatment of anterior cruciate ligament insufficiency. A retrospective matched-pair long-term follow-up. Int Orthop 2011;35:607-13.

9 Fu FH, van Eck CF, Tashman S, et al. Anatomic anterior cruciate ligament reconstruction: a changing paradigm. Knee Surg Sports Traumatol Arthrosc 2015;23:640-8.

10 Harris KP, Driban JB, Sitler MR, et al. Tibiofemoral osteoarthritis after surgical or nonsurgical treatment of anterior cruciate ligament rupture: a systematic review. J Ath/ Train 2017:52:507-17.

11 Lie MM, Risberg MA, Storheim K, et al. What's the rate of knee osteoarthritis 10 years after anterior cruciate ligament injury? an updated systematic review. $\mathrm{Br}$ J Sports Med 2019:53:1162-7.

12 Moher D, Liberati A, Tetzlaff J, et al. Preferred reporting items for systematic reviews and meta-analyses: the PRISMA statement. BMJ 2009;339:b2535.

13 Tegner Y, Lysholm J. Rating systems in the evaluation of knee ligament injuries. Clin Orthop Relat Res 1985:43-9.

14 Daniel DM, Stone ML, Sachs R, et al. Instrumented measurement of anterior knee laxity in patients with acute anterior cruciate ligament disruption. Am J Sports Med $1985 ; 13: 401-7$

15 Roos EM, Lohmander LS. The knee injury and osteoarthritis outcome score (KOOS): from joint injury to osteoarthritis. Health Qual Life Outcomes 2003;1:64. (no pagination).

16 Irrgang JJ, $\mathrm{Ho} \mathrm{H}$, Harner CD, et al. Use of the International knee documentation Committee guidelines to assess outcome following anterior cruciate ligament reconstruction. Knee Surg Sports Traumatol Arthrosc 1998;6:107-14.

17 Lysholm J, Gillquist J. Evaluation of knee ligament surgery results with special emphasis on use of a scoring scale. Am J Sports Med 1982;10:150-4.

18 Culvenor AG, Engen CN, Øiestad BE, et al. Defining the presence of radiographic knee osteoarthritis: a comparison between the Kellgren and Lawrence system and OARS atlas criteria. Knee Surg Sports Traumatol Arthrosc 2015;23:3532-9.

19 Kellgren JH, Lawrence JS. Radiological assessment of osteo-arthrosis. Ann Rheum Dis 1957; 16:494-502.

20 Hefti F, Müller W, Jakob RP, et al. Evaluation of knee ligament injuries with the IKDC form. Knee Surg Sports Traumatol Arthrosc 1993;1:226-34.

21 Altman RD, Hochberg M, Murphy WA, et al. Atlas of individual radiographic features in osteoarthritis. Osteoarthritis Cartilage 1995;3(Suppl A):3-70.

22 Downs SH, Black N. The feasibility of creating a checklist for the assessment of the methodological quality both of randomised and non-randomised studies of health care interventions. J Epidemiol Community Health 1998;52:377-84.
23 Deeks J, Dinnes J, D'Amico R, et al. Evaluating non-randomised intervention studies. Health Technol Assess 2003;7:iii-x:1-173.

24 Meuffels DE, Favejee MM, Vissers MM, et al. Ten year follow-up study comparing conservative versus operative treatment of anterior cruciate ligament ruptures. A matched-pair analysis of high level athletes. Br J Sports Med 2009;43:347-51.

25 Neuman P, Kostogiannis I, Fridén T, et al. Patellofemoral osteoarthritis 15 years after anterior cruciate ligament injury--a prospective cohort study. Osteoarthritis Cartilage 2009; 17:284-90.

26 Neuman P, Kostogiannis I, Fridén T, et al. Knee laxity after complete anterior cruciate ligament tear: a prospective study over 15 years. Scand J Med Sci Sports 2012;22:156-63.

27 Neuman P, Englund M, Kostogiannis I, et al. Prevalence of tibiofemoral osteoarthritis 15 years after nonoperative treatment of anterior cruciate ligament injury: a prospective cohort study. Am J Sports Med 2008;36:1717-25.

28 Tsoukas D, Fotopoulos V, Basdekis G, et al. No difference in osteoarthritis after surgical and non-surgical treatment of ACL-injured knees after 10 years. Knee Surg Sports Traumatol Arthrosc 2016;24:2953-9.

29 van Yperen DT, Reijman M, van Es EM, et al. Twenty-year follow-up study comparing operative versus Nonoperative treatment of anterior cruciate ligament ruptures in high-level athletes. Am J Sports Med 2018;46:1129-36.

30 Marx RG, Jones EC, Angel M, et al. Beliefs and attitudes of members of the American Academy of orthopaedic surgeons regarding the treatment of anterior cruciate ligament injury. Arthroscopy 2003;19:762-70.

31 Kujala UM, Kettunen J, Paananen $\mathrm{H}$, et al. Knee osteoarthritis in former runners, soccer players, weight lifters, and shooters. Arthritis Rheum 1995;38:539-46.

32 Øiestad BE, Holm I, Risberg MA. Return to pivoting sport after ACL reconstruction: association with osteoarthritis and knee function at the 15-year follow-up. Br J Sports Med 2018;52:1199-204.

33 Frobell RB, Lohmander LS, Roos EM. The challenge of recruiting patients with anterior cruciate ligament injury of the knee into a randomized clinical trial comparing surgical and non-surgical treatment. Contemp Clin Trials 2007;28:295-302.

34 Flosadottir V, Roos EM, Ageberg E. Muscle function is associated with future patientreported outcomes in young adults with $A C L$ injury. BMJ Open Sport Exerc Med 2016:2:e000154.

35 Cinque ME, Dornan GJ, Chahla J, et al. High rates of osteoarthritis develop after anterior cruciate ligament surgery: an analysis of 4108 patients. Am J Sports Med 2018;46:2011-9.

36 Wright RW, MARS Group. Osteoarthritis classification scales: interobserver reliability and arthroscopic correlation. J Bone Joint Surg Am 2014;96:1145-51.

37 Barker K, Lamb SE, Toye F, et al. Association between radiographic joint space narrowing, function, pain and muscle power in severe osteoarthritis of the knee. Clin Rehabil 2004;18:793-800.

38 Øiestad BE, Holm I, Risberg MA. 293 the association between radiographic knee osteoarthritis and knee symptoms function and quality of life 10-15 years after anterior cruciate ligament reconstruction. Osteoarthritis Cartilage 2010;18.

39 Sanders TL, Kremers HM, Bryan AJ, et al. Is anterior cruciate ligament reconstruction effective in preventing secondary meniscal tears and osteoarthritis? Am J Sports Med 2016;44:1699-707. 